\title{
SYNTHESIS AND LUMINESCENCE PROPERTIES OF CdPS INTERCALATED WITH RARE EARTH CATIONS
}

\author{
Rene Clement, $\dagger$ Anne Leaustic, $\dagger$ Kimberly Marney $\ddagger$ and A. H. Francis $\ddagger$ \\ †Laboratoire de Chimie Inorganique, U.R.A. 420, Bt 420, Université de Paris Sud, \\ 91405 Orsay Cedex, France \\ tDepartment of Chemistry. The University of Michigan, Ann Arbor, MI 48109, U.S.A.
}

(Received 6 July 1993; accepted 23 Sepiember 1993)

\begin{abstract}
Nd}^{3+}, \mathrm{Sm}^{3+}$ and $\mathrm{Eu}^{3+}$ have been intercalated by a cation exchange procedure into the layered lattice of $\mathrm{CdPS}_{3}$. X-ray diffraction measurements of the interlamellar expansion demonstrate that the cations initially occupy the interlamellar planes and are solvated by a water bilayer. Dehydration of the lattice occurs at $120^{\circ} \mathrm{C}$ and is accompanied by a substantial decrease in the interlamellar spacing. Analysis of the host lattice photoluminescence and i.r. spectra indicate cadmium cation vacancies are created in the host lattice to balance the intercalated charge. The crystal field effects on the rare earth cation photoluminescence indicate that the cations have entered the intralamellar cation vacancies to form a complex defect center with $\mathrm{C}_{2}$ or lower symmetry. It is proposed that replacing dipositive $\mathrm{Cd}^{2+}$ cations with $\mathrm{RE}^{3+}$ cations creates a characteristic complex involving two $\mathrm{RE}^{3+}$ cations and a lattice vacancy, $\mathrm{V}^{2-}$.
\end{abstract}

Keywords: Rare earth, luminescence, vacancy, layered, chalcogenide, transition metal.

\section{INTRODUCTION}

Transition metal trichalcogenides, $\mathbf{M}_{2} \mathbf{P}_{2} \mathbf{S}_{6}$, where $\mathbf{M}$ is a divalent transition metal, form a broad class of lamellar semiconductors that are structurally related to the transition metal dichalcogenides [1,2]. The $\mathbf{M}_{2} \mathbf{P}_{2} \mathbf{S}_{6}$ structure is built of slabs constructed from face-sharing $\mathrm{MS}_{6}$ and $\mathrm{P}_{2} \mathrm{~S}_{6}$ octahedra. Adjacent slabs are held together by relatively weak van der Waal forces [3] to form easily accessible interstitial spaces called "van der Waals gaps" (vWG). When crystalline $\mathrm{MPS}_{3}$ materials are placed in contact with solutions containing mono-, di- or tri-valent cations, the solute cations enter the vWG and lattice cations are in turn displaced into the solution [4]. Figure 1 is a schematic of the cation exchange process in these materials. It is supposed that solute cations enter the lattice via the weakly bonded interlamellar spaces and that lattice metal cations depart via the same route. This process involves a complex and interesting heterogeneous equilibrium between solvated cationic species in solution with those in the VWG and in regular lattice sites.

We have previously examined this process [5] by analysis of the EPR spectra of paramagnetic, dipositive transition metal cations (Mn, Co) as they exchange with the diamagnetic, dipositive cations $(\mathrm{Zn}, \mathrm{Cd})$. In these studies it was shown that the guest cations $\left(\mathrm{G}^{2+}\right)$ can occupy vacancies created by displaced lattice metal ions $\left(\mathrm{M}^{2+}\right)$ to form substitu- tional centers. However, when the lattice cations are replaced by tripositive intercalate cations, a charge imbalance is created that must be locally compensated. The requirement for charge compensation leads to the formation of more complex centers involving the clustering of ions and vacancies.

In the present work we have employed photoluminescence (PL) spectroscopy to examine the structure of centers formed by the intercalation of tri-positive rare earth (RE) cations into $\mathrm{CdPS}_{3}$. The $\mathrm{RE}^{3+}$ cations retain many of their atomic-like properties in the lattice and sharp, well-resolved PL spectra characteristic of $f$-electron transitions are observed. The details of the crystal coordination environment can be derived from the relatively weak crystal field effects observed in the spectra [6].

\section{EXPERIMENTAL}

\section{Sample preparation}

Host crystals of CdPS, were grown using a modified version of the iodine vapor transport method [7]. Stoichiometric amounts of cadmium, sulfur and red phosphorus were mixed together and placed in a quartz tube together with a small amount of $\mathrm{I}_{2}$. The tube was sealed under vacuum, heated to $680^{\circ} \mathrm{C}$ and maintained at this temperature for 1 week.

Intercalation of $\mathrm{Nd}^{3+}, \mathrm{Sm}^{3+}$ or $\mathrm{Eu}^{3+}$ into $\mathrm{CdPS}_{3}$ was accomplished by a two-step process involving an 


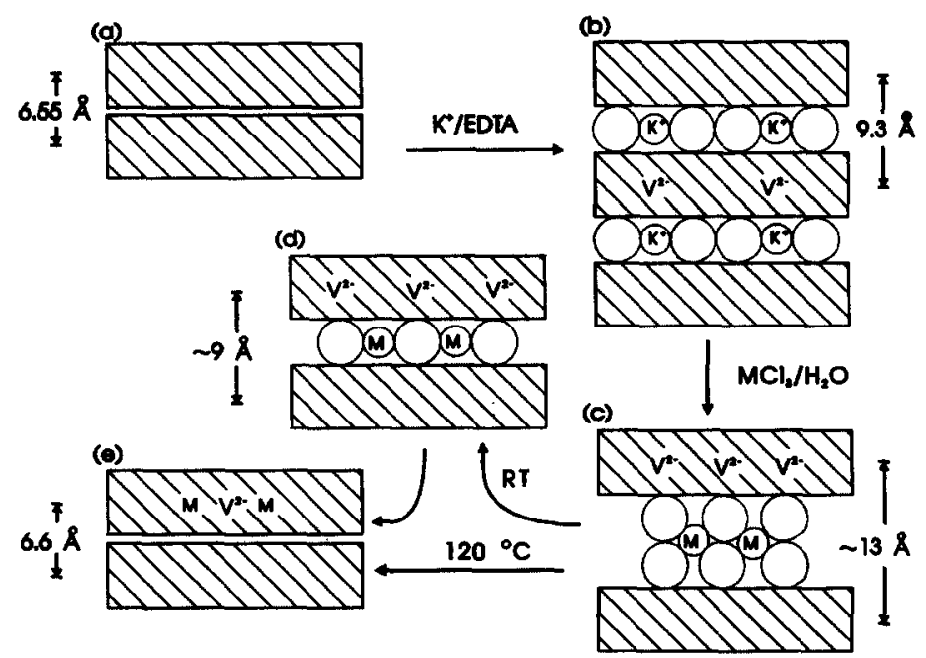

Fig. 1. Schematic representation of the cation-exchange intercalation reaction in $\mathbf{M}_{2} \mathbf{P}_{2} \mathrm{~S}_{6}$ lattices. (a) Pristine $\mathrm{CdPS}_{3}$, (b) $\mathrm{CdPS}_{3}: \mathrm{K}^{+}$, (c) $\mathrm{CdPS}_{3}: \mathrm{RE}^{3+}$, (d) $\mathrm{CdPS}_{3}: \mathrm{Eu}^{3+}$ (partially dehydrated), (e) $\mathrm{CdPS}_{3}: \mathrm{RE}^{3+}$ (dehydrated).

initial exchange with $\mathrm{K}^{+}$followed by exchange with the $\mathrm{RE}^{3+}$ cations (see Fig. 1). Vapor grown $\mathrm{CdPS}_{3}$ crystals were soaked for $24 \mathrm{~h}$ in $10 \mathrm{ml}$ of a basic potassium bicarbonate buffer system $\left(0.1 \mathrm{~g} \mathrm{~K}_{2} \mathrm{CO}_{3}\right.$ and $0.1 \mathrm{~g} \mathrm{KHCO}_{3}$ ) with $1 \mathrm{~g} \mathrm{KCl}$ and $0.1 \mathrm{M}$ EDTA. The EDTA was included to chelate $\mathrm{Cd}^{2+}$ and enhance the cation exchange reaction. After the reaction was complete, the crystals were rinsed with $\mathrm{H}_{2} \mathrm{O}$ and air dried.

$\mathrm{RE}^{3+}$ cations were introduced by soaking the $\mathrm{K}^{+}$ exchanged crystals in approximately $500 \mathrm{mg}$ of the rare earth salt (RE) $\mathrm{Cl}_{3} \cdot 6 \mathrm{H}_{2} \mathrm{O}$ dissolved in $10 \mathrm{ml}$ of water for $24 \mathrm{~h}$ at $25^{\circ} \mathrm{C}$. The crystals were rinsed with $\mathrm{H}_{2} \mathrm{O}$ and air dried. Elemental analyses of the compounds that were prepared and examined are given in Table 1.

The charge deficit created by the removal of $\mathrm{Cd}^{2+}$ ions is not completely compensated by the uptake of $\mathrm{RE}^{3+}$. As discussed below, the substitution of tripositive cations for dipositive cations requires a complex charge compensation mechanism that may involve retention of $\mathrm{K}^{+}$or loss of phosphorus as a $\mathrm{P}^{4+}$ cation.
The materials are adequately described by the following general, charge-balanced formula,

$$
\mathrm{Cd}_{1-3 x / 2-y / 2} \mathbf{P}_{1-3 x / 4-y / 4} \mathbf{S}_{3}(\mathrm{RE})_{x} \mathrm{~K}_{y}
$$

that is in reasonable agreement with the observed stoichiometries. X-ray diffractograms were obtained at several temperatures between $20^{\circ} \mathrm{C}$ and $300^{\circ} \mathrm{C}$ using a McPherson powder diffractometer with a $\mathrm{CuK}_{\mathrm{a}} \quad(\lambda=1.5405 \AA)$ source. Basal spacing measurements were made by monitoring the $\{001\}$ peak with a fast scan $\left(120^{\circ} \mathrm{h}^{-1}\right)$ from $2 \theta$ values of $2^{\circ}$ to $15^{\circ}$. A $9.3 \AA$ basal spacing was observed for the potassium cation exchanged crystals and the $\mathrm{Eu}^{3+}$ exchanged crystals after dehydration at $50^{\circ} \mathrm{C}$. The basal spacings of all freshly prepared RE samples were $\sim 13 \AA$, but upon standing for one month approximately half of the material converted to a dehydrated form with a $\sim 6.6 \AA$ basal spacing. All crystals underwent rapid conversion to the $\sim 6.6 \AA$ basal spacing when dehydrated by heating to $120^{\circ} \mathrm{C}$ under vacuum. The loss of all water at $120^{\circ} \mathrm{C}$ was confirmed by TGA.

Table 1. Elemental analyses of cation exchanged lattices and lattice basal spacings

\begin{tabular}{lcl}
\hline Compound & $\begin{array}{c}\text { Basal } \\
\text { spacing }(\AA)\end{array}$ & \multicolumn{1}{c}{ Comment } \\
\hline $\mathrm{CdPS}_{3}$ & 6.55 & Host lattice \\
$\mathrm{Cd}_{0.78} \mathrm{P}_{1.00} \mathrm{~S}_{3} \mathrm{Eu}_{0.092} \mathrm{~K}_{0.020}\left(\mathrm{H}_{2} \mathrm{O}\right)_{\sim 4}$ & 12.9 & Hydrated, bilayer \\
$\mathrm{Cd}_{0.78} \mathrm{P}_{1.00} \mathrm{~S}_{3} \mathrm{Eu}_{0.092} \mathrm{~K}_{0.020}\left(\mathrm{H}_{2} \mathrm{O}\right)_{\sim 2}$ & 9.15 & Hydrated, monolayer \\
$\mathrm{Cd}_{0.78} \mathrm{P}_{1.00} \mathrm{~S}_{3} \mathrm{Eu}_{0.092} \mathrm{~K}_{0.020}$ & 8.10 & Dehydrated, interlamellar $\mathrm{Eu}$ \\
$\mathrm{Cd}_{0.78} \mathrm{P}_{1.00} \mathrm{~S}_{3} \mathrm{Eu}_{0.092} \mathrm{~K}_{0.020}$ & 6.6 & Dehydrated, intralamellar, Eu \\
$\mathrm{Cd}_{0.84} \mathrm{P}_{0.97} \mathrm{~S}_{3} \mathrm{Nd}_{0.12} \mathrm{~K}_{0.07}\left[\mathrm{H}_{2} \mathrm{O}\right]_{\sim 3}$ & 13.42 & Hydrated, bilayer \\
$\mathrm{Cd}_{0.84} \mathrm{P}_{0.97} \mathrm{~S}_{3} \mathrm{Nd}_{0.12} \mathrm{~K}_{0.07}$ & 6.6 & Dehydrated, interlamellar $\mathrm{Nd}$ \\
$\mathrm{Cd}_{0.85} \mathrm{P}_{0.96} \mathrm{~S}_{3} \mathrm{Sm}_{0.12} \mathrm{~K}_{0.08}\left[\mathrm{H}_{2} \mathrm{O}\right]_{\sim 3}$ & 13.4 & Hydrated, bilayer \\
$\mathrm{Cd}_{0.85} \mathrm{P}_{0.96} \mathrm{~S}_{3} \mathrm{Sm}_{0.12} \mathrm{~K}_{0.08}$ & 6.6 & Dehydrated, intralamellar Sm \\
\hline
\end{tabular}




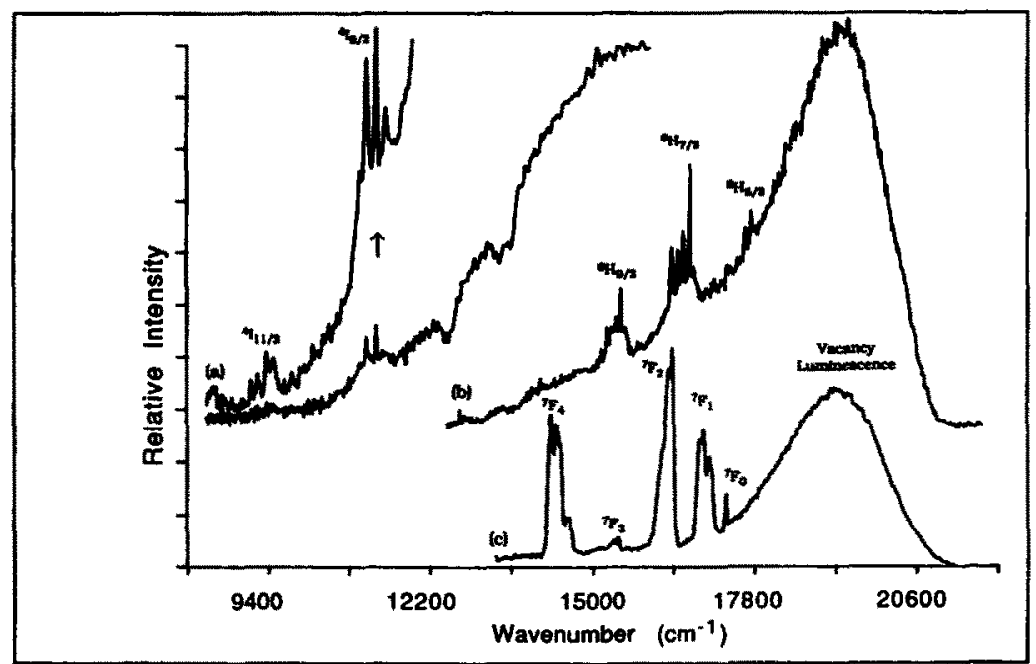

Fig. 2. Photoluminescence spectra of $\mathrm{CdPS}_{3}$ exchanged with (a) $\mathrm{Nd}^{3+}$ (hydrated), recorded at $77 \mathrm{~K}$, (b) $\mathrm{Sm}^{3+}$ (dehydrated) at $4.2 \mathrm{~K}$ and (c) $\mathrm{Eu}^{3+}$ (dehydrated) at $4.2 \mathrm{~K}$.

\section{Photoluminescence ( $P L$ ) spectra}

PL spectra were recorded at $77 \mathrm{~K}$ and $4 \mathrm{~K}$ by placing samples in thermal equilibrium with normal boiling liquid nitrogen or liquid helium in a Janis 10DT cryostat. PL was excited either by broad-band u.v. radiation or by selective wavelength excitation using a $5 \mathrm{~W}$ (all lines) Coherent Argon ion laser. The broad band excitation source was a $1 \mathrm{~kW}$ highpressure xenon arc lamp whose output was filtered through a $\mathrm{CuSO}_{4}$ solution filter and a Corning glass CS7-54 filter. This combination provides wavelengths between about 3300 and $4000 \AA$.

The luminescence was passed through a Corning CS3-70 filter, dispersed with a $f / 6.7$ scanning monochromator and detected with either a cooled Hamamatsu R375 or R1767 photomultiplier tube with an extended $S 1$ response. Luminescence bands of wavelength longer than about $12,500 \AA$ could not be observed due to lack of photomultiplier sensitivity

Table 2. Spectral assignments for $\mathrm{Nd}^{3+}$ intercalated $\mathrm{CdPS}_{3}$

\begin{tabular}{lccc}
\hline $\begin{array}{l}\text { Frequency } \\
\left(\mathrm{cm}^{-1}\right) \dagger\end{array}$ & $\begin{array}{c}\text { Relative } \\
\text { intensity } \ddagger\end{array}$ & $\begin{array}{c}\text { Intramultiplet } \\
\text { interval }\end{array}$ & Assignments \\
\hline$(19,142)$ & $\mathrm{m}$ & & $\left\{{ }^{2} G_{9 / 2},{ }^{4} G_{9 / 2}\right\} \leftarrow{ }^{4} I_{9 / 2}$ \\
$(17,265)$ & $\mathrm{s}$ & & $\left\{{ }^{2} G_{7 / 2},{ }^{4} G_{5 / 2}\right\} \leftarrow$ \\
$(16,077)$ & $\mathrm{m}$ & & ${ }^{2} H_{1 / 2} \leftarrow$ \\
$(13,514)$ & $\mathrm{m}$ & & $\left\{{ }^{4} F_{7 / 2},{ }^{4} S_{3 / 2}\right\} \leftarrow$ \\
$(12,579)$ & $\mathrm{m}$ & & $\left\{{ }^{2} H_{9 / 2},{ }^{4} F_{5 / 2}\right\} \leftarrow$ \\
11,417 & $\mathrm{~m}$ & 0 & ${ }^{4} F_{3 / 2} \rightarrow{ }^{4} I_{9 / 2}$ \\
11,254 & $\mathrm{~s}$ & 163 & ${ }^{4} F_{3 / 2} \rightarrow{ }^{4} I_{9 / 2}$ \\
11,090 & $\mathrm{~s}$ & 327 & ${ }^{4} F_{3 / 2} \rightarrow{ }^{4} I_{9 / 2}$ \\
10,954 & $\mathrm{w}$ & 463 & ${ }^{4} F_{3 / 2} \rightarrow{ }^{4} I_{9 / 2}$ \\
10,913 & $\mathrm{w}$ & 504 & ${ }^{4} F_{3 / 2} \rightarrow{ }^{4} I_{9 / 2}$ \\
9477 & $\mathrm{~m}$ & 0 & ${ }^{4} F_{3 / 2} \rightarrow{ }^{4} I_{9 / 2}$ \\
9360 & $\mathrm{~m}$ & 117 & ${ }^{4} F_{3 / 2} \rightarrow{ }^{4} I_{9 / 2}$ \\
\hline
\end{tabular}

†rackets denote absorption bands.

$\ddagger \mathbf{w}=$ weak; $\mathbf{m}=$ medium; $s=$ strong. beyond this wavelength. The spectra are not corrected for the photomultiplier response.

\section{RESULTS}

\section{$\mathrm{CdPS}_{3}: \mathrm{Nd}^{3+}$}

The luminescence of $\mathrm{Nd}^{3+}$ exchanged samples was excited using the $5145 \AA$ line of the Argon ion laser. Spectra were recorded at $77 \mathrm{~K}$ since no appreciable neodymium luminescence was detected at $4 \mathrm{~K}$. The PL spectra exhibit the broad band luminescence between approximately $5000-8000 \AA$, characteristic of lattice cation vacancies. Additional weak, sharp-line $\mathrm{Nd}^{3+}$ luminescence is also observed (see Fig. 2a). The vacancy luminescence is partially reabsorbed by $\mathrm{Nd}^{3+}$ giving rise to several regions of sharp-line absorption. The analysis of the $\mathrm{Nd}^{3+}$ spectrum is given in Table 2. The general positions of the J-multiplets is typical of $\mathrm{Nd}^{3+}$ in many crystal lattices [8].

Table 3. Spectral assignments for $\mathrm{Sm}^{3+}$ intercalated $\mathrm{CdPS}_{3}$

\begin{tabular}{lccl}
\hline $\begin{array}{l}\text { Frequency } \\
\text { (cm }{ }^{1} \text { ) }\end{array}$ & $\begin{array}{c}\text { Relative } \\
\text { intensity } \dagger\end{array}$ & $\begin{array}{c}\text { Intramultiplet } \\
\text { intervals }\end{array}$ & Assignments \\
\hline 17,724 & $\mathrm{w}$ & 0 & ${ }^{4} G_{5 / 2} \rightarrow{ }^{6} H_{5 / 2}$ \\
17,659 & $\mathrm{~m}$ & -65 & ${ }^{4} G_{5 / 2} \rightarrow{ }^{6} H_{5 / 2}$ \\
17,562 & $\mathrm{~m}$ & -162 & ${ }^{4} G_{5 / 2} \rightarrow{ }^{6} H_{5 / 2}$ \\
17,311 & $\mathrm{w}$ & & ${ }^{4} G_{5 / 2} \rightarrow{ }^{6} H_{5 / 2}$ \\
17,245 & $\mathrm{w}$ & & ${ }^{4} G_{5 / 2} \rightarrow{ }^{6} H_{5 / 2}$ \\
16,703 & $\mathrm{w}$ & +86 & \\
16,617 & $\mathrm{~s}$ & 0 & ${ }^{4} G_{5 / 2} \rightarrow{ }^{6} H_{7 / 2}$ \\
16,503 & $\mathrm{~m}$ & -114 & ${ }^{4} G_{5 / 2} \rightarrow{ }^{6} H_{7 / 2}$ \\
16,405 & $\mathrm{~m}$ & -212 & ${ }^{4} G_{5 / 2} \rightarrow{ }^{6} H_{7 / 2}$ \\
16,299 & $\mathrm{~m}$ & -318 & ${ }^{4} G_{5 / 2} \rightarrow{ }^{6} H_{7 / 2}$ \\
15,478 & $\mathrm{w}$ & +69 & \\
15,409 & $\mathrm{~s}$ & 0 & ${ }^{4} G_{5 / 2} \rightarrow{ }^{6} H_{9 / 2}$ \\
15,331 & $\mathrm{~m}$ & -78 & ${ }^{4} G_{5 / 2} \rightarrow{ }^{6} H_{9 / 2}$ \\
15,252 & $\mathrm{~m}$ & -157 & ${ }^{4} G_{5 / 2} \rightarrow{ }^{6} H_{9 / 2}$ \\
15,176 & $\mathrm{~m}$ & -233 & ${ }^{4} G_{5 / 2} \rightarrow{ }^{6} H_{9 / 2}$ \\
15,079 & $\mathrm{~m}$ & -330 & ${ }^{4} G_{5 / 2} \rightarrow{ }^{6} H_{9 / 2}$ \\
\hline
\end{tabular}

$\uparrow \mathrm{w}=$ weak; $\mathrm{m}=$ medium; $\mathrm{s}=$ strong. 

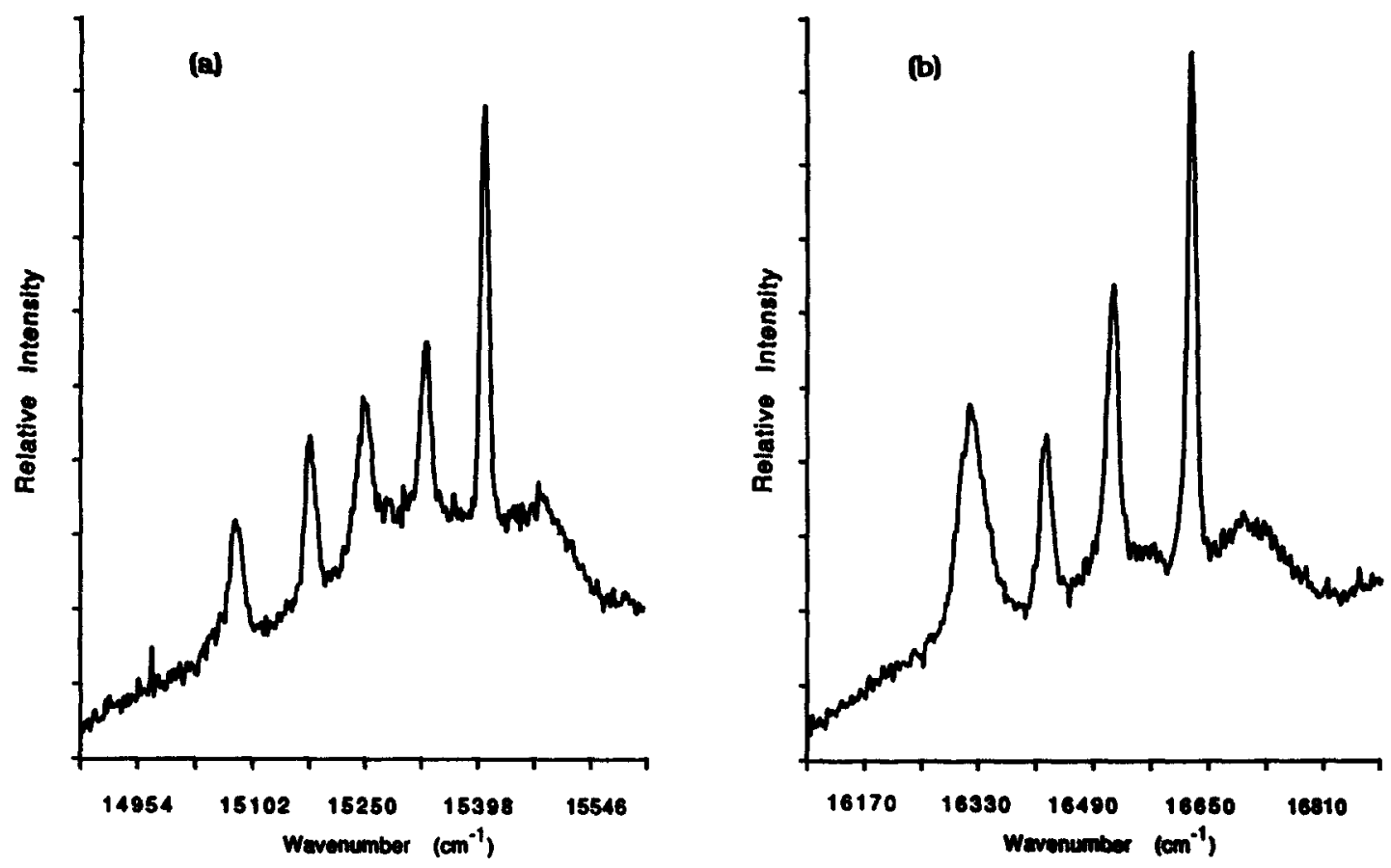

Fig. 3. Spectra of the $\mathrm{Sm}^{3+}$ (a) ${ }^{4} G_{5 / 2} \rightarrow{ }^{6} H_{9 / 2}$, (b) ${ }^{4} G_{5 / 2} \rightarrow{ }^{6} H_{7 / 2}$ transitions.

\section{$\mathrm{CdPS}_{3}: \mathrm{Sm}^{3+}$}

PL spectra were recorded at $4 \mathrm{~K}$ for both the hydrated and dehydrated phases. Luminescence (see Fig. 2b) was stimulated most efficiently by the broadband excitation source and the analysis is given in Table 3. The general positions of the J-multiplets are similar to those in the literature [9]. Spectra of selected multiplets are shown in greater detail in Fig. 3. The effect of dehydration of the PL spectrum is illustrated in Fig. 5. Although the spectrum of the hydrated phase is weaker, the J-multiplet appears to be split into fewer components by the crystal field.

\section{$\mathrm{CdPS}_{3}: \mathrm{Eu}^{3+}$}

Of the several europium-containing compounds, only the dehydrated phase exhibits photoluminescence. When the samples were cooled to $4 \mathrm{~K}$ and illuminated with broad-band u.v. radiation, the PL spectrum illustrated in Fig. 2c was obtained. The analysis in Table 4 is typical of $\mathrm{Eu}^{3+}$ in crystalline lattices [10].

\section{DISCUSSION}

\section{Cation exchange chemistry}

Cation exchange produces a 2.8 and a $6.8 \AA$ increase in the basal spacing of the host lattice. These increases approximately correspond to the RE cation diameter plus one or two van der Waals diameter of water $(2.8 \AA)$. The monolayer and bilayer solvation models shown schematically in Fig. 1 are consistent with the observed increase in basal spacing. In fact, analogous behaviour has been observed when $\mathrm{TaS}_{2}$ and $\mathrm{TiS}_{2}$ are intercalated with hydrated alkali metal cations. These much studied dichalcogenides have a lamellar structure similar to $\mathrm{CdPS}_{3}$. In the cation intercalated compounds of $\mathrm{TaS}_{2}$ and $\mathrm{TiS}_{2}$, the basal spacing and amount of water intercalated with the cation is governed by the intercalated cation's chargeto-radius ratio. A ratio less than one results in a monolayer of water while a ratio greater than one produces a bilayer of water [11]. This charge-toradius ratio rule appears to apply to the cation exchanged $\mathrm{CdPS}_{3}$ compounds where a ratio greater than one for $\mathrm{Nd}^{3+}, \mathrm{Sm}^{3+}$ and $\mathrm{Eu}^{3+}$ results in a water solvation bilayer. The basal spacing of the hydrated phases returns to nearly that of the host lattice upon dehydration. This suggests that the RE cations have entered $\mathrm{Cd}$ vacancies. The ionic radii of $\mathrm{Nd}^{3+}$ $(0.995 \AA), \mathrm{Sm}^{3+}(0.964 \AA)$ and $\mathrm{Eu}^{3+}(0.950 \AA)$ allow them to insert into the $\mathrm{Cd}^{2+}$ vacancy $(\sim 1.1 \AA)$.

The substitution of $\mathrm{RE}^{3+}$ for $\mathrm{Cd}^{2+}$ may lead to the formation of complex point defect centers in which ions and vacancies associate in order to achieve charge neutrality and reduce crystal lattice energy. Although there are examples of charge compensation by distant ions or vacancies, association is commonly observed [12]. A simple cluster of two $\mathrm{RE}^{3+}$ and a $\mathrm{V}^{2}$ form a $\mathrm{M}-\mathrm{V}-\mathrm{M}$ neutral center. If a monopositive 
b
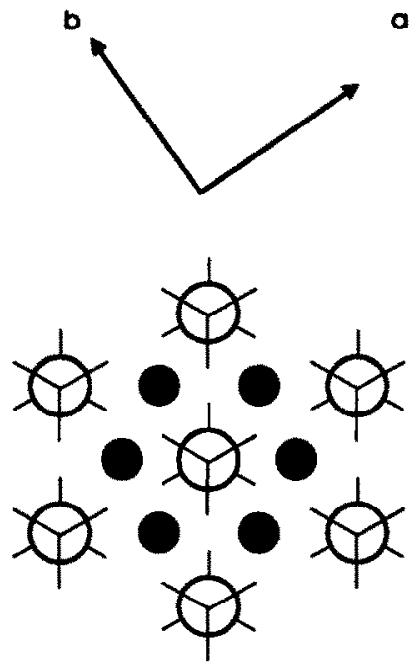

(a)

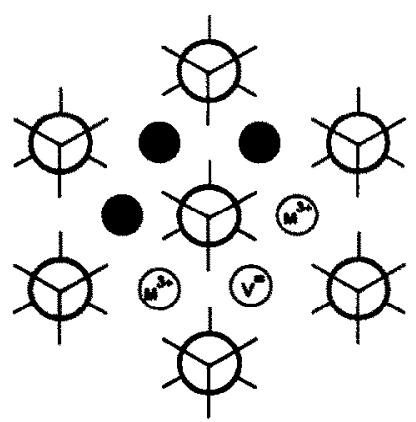

(c)
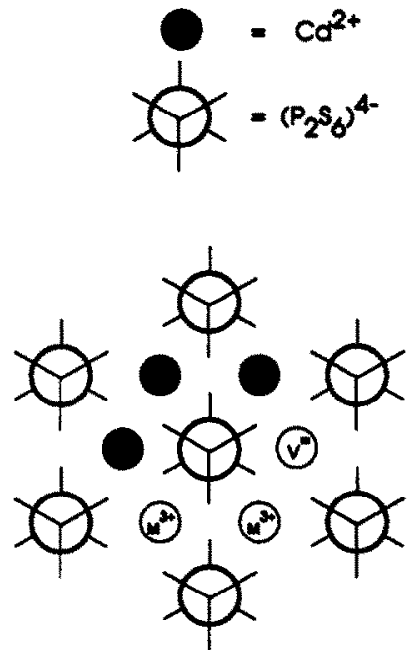

(b)

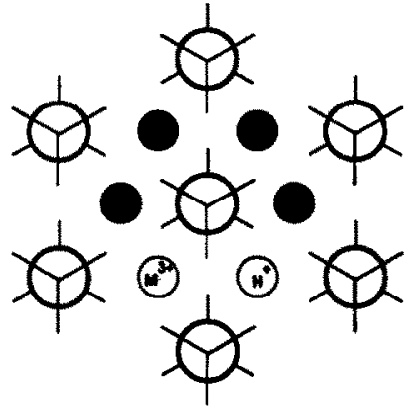

(d)

Fig. 4. Site models. (a) The host $\mathrm{CdPS}_{3}$ lattice viewed perpendicular to the $a b$-plane. (b-c) Two arrangements of a vacancy and rare earth ions producing sites of $C_{2}$ symmetry in the nearest coordination shell.

Table 4. Spectral assignments for $\mathrm{Eu}^{3+}$ intercalated $\mathrm{CdPS}_{3}$

\begin{tabular}{lccc}
\hline $\begin{array}{l}\text { Frequency } \\
\left(\mathrm{cm}^{-1}\right)\end{array}$ & $\begin{array}{c}\text { Relative } \\
\text { intensity }\end{array}$ & $\begin{array}{c}\text { Intramultiplet } \\
\text { interval }\end{array}$ & Assignment \\
\hline 17,313 & $\mathrm{~m}$ & & ${ }^{5} D_{0} \rightarrow{ }^{7} F_{0}$ \\
17,018 & $\mathrm{~m}$ & 0 & ${ }^{5} D_{0} \rightarrow{ }^{7} F_{1}$ \\
16,915 & $\mathrm{~s}$ & -103 & ${ }^{5} D_{0} \rightarrow{ }^{7} F_{1}$ \\
16,858 & $\mathrm{~s}$ & -160 & ${ }^{5} D_{0} \rightarrow{ }^{7} F_{1}$ \\
16,383 & $\mathrm{~s}$ & 0 & \\
16,319 & $\mathrm{~s}$ & -64 & ${ }^{5} D_{0} \rightarrow{ }^{7} F_{2}$ \\
16,213 & $\mathrm{~s}$ & -170 & ${ }^{5} D_{0} \rightarrow{ }^{7} F_{2}$ \\
& & & \\
15,423 & $\mathrm{w}$ & 0 & ${ }^{5} D_{0}{ }^{5} D_{0} \rightarrow{ }^{7} F_{3}$ \\
15,366 & $\mathrm{w}$ & -57 & ${ }^{5} D_{0} \rightarrow{ }^{7} F_{3}$ \\
& & & \\
14,594 & $\mathrm{~m}$ & 0 & ${ }^{5} D_{0} \rightarrow{ }^{7} F_{4}$ \\
14,560 & $\mathrm{~m}$ & -34 & ${ }^{5} D_{0} \rightarrow{ }^{7} F_{4}$ \\
14,434 & $\mathrm{~s}$ & -160 & ${ }^{5} D_{0} \rightarrow{ }^{7} F_{4}$ \\
14,376 & $\mathrm{~s}$ & -218 & ${ }^{5} D_{0} \rightarrow{ }^{7} F_{4}$ \\
14,278 & $\mathrm{~s}$ & -316 & ${ }^{5} D_{0} \rightarrow{ }^{7} F_{4}$ \\
\hline
\end{tabular}

positive ion ( $\mathbf{M}^{\prime}$ ) is present, a $\mathbf{M}-\mathbf{M}^{\prime}$ center can be formed and a lattice vacancy is not required. Monopositive ions, $\mathrm{K}^{+}$and $\mathrm{H}^{+}$, are present in the aqueous cation exchange solution and could participate the exchange scheme, however, the $\mathrm{M}-\mathrm{V}-\mathrm{M}$ structure is preferred since the PL and i.r. data reveal the presence of vacancies. The hexagonal arrangement of the intralamellar cation sites results in the defect structures illustrated in Fig. 4a-d. The structures shown offer the lowest defect volumes and are believed to be the most probable.

The clustering of cations and vacancies is similar to that reported for $\mathrm{RE}$ ions in $\mathrm{AMX}_{3}$ lattices $\mathrm{A}=$ alkali metal; $\mathbf{M}=\mathbf{M g}, \mathbf{C d}$ ). $\mathbf{R E}^{3+}$ cations can be incorporated into the divalent metal sites of the $\mathrm{AMX}_{3}$ lattice by replacing $3 \mathrm{M}^{2+}$ with $2 \mathrm{M}^{3+}$ and a vacancy $\mathrm{V}^{2-}$. If a source of monopositive ions is available, charge neutrality is satisfied by the exchange of one $\mathrm{M}^{3+}$ for a $\mathbf{M}^{2+}-\mathbf{M}^{+}$pair [13]. 


\section{$\mathrm{CdPS}_{3}$ photoluminescence}

Well-annealed crystals of the $\mathrm{CdPS}_{3}$ host lattice are only weakly luminescent. However, after treatment with aqueous solutions containing solute cations $\left(\mathrm{G}^{n+}\right)$, the $\mathrm{CdPS}_{3}$ lattices exhibit an intense, broad luminescence between approximately $4600-6000 \AA$ [14]. The luminescence is long lived $(65 \mu \mathrm{s})$ and originates from cation vacancy sites created by cation exchange according to the reaction,

$$
\mathrm{CdPS}_{3}+y \mathrm{G}^{n+} \leftrightarrow \mathrm{Cd}_{1-n y / 2} \mathrm{G}_{y} \mathrm{PS}_{3}+(n y / 2) \mathrm{Cd}^{2+} .
$$

If $n \leqslant 2$, the number of vacancies created is equal to or less than the number of intercalated cations. Therefore, if the ionic radius permits, the guest cations may enter the lattice substitutionally for $\mathrm{Cd}^{2+}$ filling all of the lattice vacancies. If $n>2$ the number of vacancies created is greater than the number of guest cations and some vacancies must remain. When $\mathrm{CdPS}_{3}$ is exchanged with $\mathrm{RE}^{3+}$ cations, the vacancy PL spectrum remains even after heat treatment to shift the interlamellar $\mathrm{RE}$ cations into the $\mathrm{Cd}$ vacancies, confirming that uncompensated vacancies remain for $n>2$.

The excited state of the vacancy defect center is a spin triplet and has been examined by ODMR spectroscopy. The spectrum arises from two electrons trapped at the cation vacancy forming a $\mathrm{V}^{2-}$ center. The presence of cation vacancies is also indicated by changes in the i.r. spectrum. The $\mathrm{PS}_{3}$ asymmetric stretch, observed at $570 \mathrm{~cm}^{-1}$ in the i.r. spectrum of $\mathrm{CdPS}_{3}$, is split into two components at $605 \mathrm{~cm}^{-1}$ and $555 \mathrm{~cm}^{-1}$. It is well established [15] that this effect in the i.r. spectrum is caused by the creation of intralamellar vacancies in MPS $_{3}$ intercalates.

\section{$\mathbf{R E}^{3+}$ luminescence}

Additional luminescence bands due to RE cations appear superimposed on the $\mathrm{V}^{2}$ - luminescence. The $\mathrm{RE}^{3+}$ luminescence arises from intra-configurational, $4 f$-electron transitions and is typically sharp and well resolved since the $f$-electron transitions are not strongly perturbed by the lattice. The luminescence studied in this work is derived from the following $\mathrm{RE}^{3+}$ free-ion transitions:

$$
\begin{aligned}
\operatorname{Nd}^{3+}\left(4 f^{3}\right):{ }^{4} F_{3 / 2} & \rightarrow{ }^{4} I_{J} \quad(J=15 / 2,13 / 2,11 / 2,9 / 2) \\
\operatorname{SM}^{3+}\left(4 f^{5}\right):{ }^{4} G_{5 / 2} & \rightarrow{ }^{6} H_{J} \\
(J & =15 / 2,13 / 2,11 / 2,9 / 2,7 / 2,5 / 2) \\
\operatorname{Eu}^{3+}\left(4 f^{6}\right):{ }^{5} D_{0} & \rightarrow{ }^{7} F_{J} \quad(J=0,1,2,3,4,5,6) .
\end{aligned}
$$

In each case the emitting state is the lowest level of the first excited term. Only the $J$-states of the ground term shown in bold were observed. The remaining transitions are unobservable due either to host lattice emission/absorption or because they lie beyond the long wavelength limit of the phototube.

Due to the small spatial extent of the $f$-orbitals, the RE transitions are only weakly perturbed by the crystalline lattice. As a result, the frequency of transitions terminating on the $J$-states of the ground state term are determined chiefly by intra-atomic effects (spin-orbit and coulombic interactions). The detailed splitting and intensity patterns within each multiplet, however, are sensitive to both the strength and symmetry of the crystal field. The crystal field interaction has two primary effects: it partially or completely removes the degeneracy of the free ion $J$-states into a number of $\left|M_{J}\right|$ levels determined by the site symmetry of the ion, and it changes the relative intensity of the free-ion transitions.

It is possible to calculate the magnitude of the crystal field splitting using a variety of parameterizations of the crystal field. However, it is generally unnecessary to carry out these calculations in order to obtain the site symmetry and a qualitative estimate of the strength of the crystal field. For these purposes, the relative intensity and splitting of different $J$ multiplets can be correlated with the site symmetry of the RE ion. In the present study, the rhombic (or lower) symmetry of the defect center produces a splitting of each state into $2 J+1$ levels for integral $J\left(\mathrm{Eu}^{3+}\right)$ or $J+1 / 2$ levels for half-integral $J\left(\mathrm{Nd}^{3+}\right.$, $\mathrm{Sm}^{3+}$ ). Table 5 [16] gives the number of electric dipole allowed transitions predicted for $\mathrm{Eu}^{3+}$ in a variety of site symmetries.

\section{Coordination geometry}

The PL spectrum of $\mathrm{CdPS}_{3}$ : Eu yields considerable information about the coordination environment of intralamellar $\mathrm{Eu}^{3+}$ in the dehydrated phase. Although $4 f \leftrightarrow 4 f$ transitions are electric dipole forbidden for the free ion, the spectra of $\mathrm{Eu}^{3+}$ in crystalline lattices usually consist of electric dipole transitions induced by odd parity terms in the crystal field. The number of allowed electric dipole allowed transitions for a variety of site symmetries is given in Table 5. For the $4 f^{6}$ configuration, electric dipole transitions from the ${ }^{5} D_{0}$ level and odd $J$ levels of ${ }^{7} F_{J}$ are not strongly induced by the crystal field [17]. Thus, the transitions ${ }^{5} D_{0} \rightarrow{ }^{7} F_{\mathrm{J}^{\prime}} \quad(J=1,3,5)$ are expected to be weaker than the transitions

Table 5. Theoretical number of electric dipole allowed components of the ${ }^{5} D_{0} \leftrightarrow^{7} F_{j}$ transitions for low symmetries

\begin{tabular}{lcccccc}
\hline Transition & $O_{\mathrm{h}}$ & $C_{3 \mathrm{~h}}$ & $C_{3 \mathrm{v}}$ & $C_{2 \mathrm{~h}}$ & $C_{2 \mathrm{v}}$ & $C_{2}$ \\
\hline${ }^{5} D_{0} \leftrightarrow{ }^{7} F_{0}$ & - & - & 1 & $\overline{3}$ & 1 & 1 \\
${ }^{5} D_{0} \leftrightarrow{ }^{7} F_{1}$ & 1 & 2 & 2 & 3 & 3 & 3 \\
${ }^{5} D_{0} \leftrightarrow{ }^{7} F_{2}$ & - & 1 & 3 & - & 4 & 5 \\
${ }^{5} D_{0} \leftrightarrow{ }^{7} F_{3}$ & 1 & 2 & 4 & 7 & 6 & 7 \\
\hline
\end{tabular}


${ }^{5} D_{0} \rightarrow(J=2,4,6)$. The ${ }^{5} D_{0} \rightarrow{ }^{7} F_{0}$ transition is forbidden in centrosymmetric sites.

The appearance of a strong, single ${ }^{5} D_{0} \rightarrow{ }^{7} F_{0}$ transition (Fig. 2c) reveals immediately that $\mathrm{Eu}^{3+}$ resides in a site without inversion symmetry. An examination of the spectra of $\mathrm{Eu}^{3+}$ in many crystalline lattices indicates that the intensity of this transition is significant in all compounds with $C_{3 \mathrm{v}}$ or lower symmetry, whereas in others it is much weaker or absent [18]. Moreover, an intense transition is usually indicative of a strong crystal field. We also conclude that only a single type of low-symmetry emissive site is present since only a single ${ }^{5} D_{0} \rightarrow{ }^{7} F_{0}$ transition is observed. If several emissive sites were occupied, this transition should give rise to lines for each site, shifted by the local crystal field.

This conclusion is supported by the crystal field splitting of the $J=1, \mathrm{~T} 2$ transitions. It is seen that the degeneracy of each $J$-state is completely lifted by the low site symmetry. For the best resolved multiplets, the number of components observed is equal to the maximum degeneracy. Other multiplets exhibit fewer than the maximum number of components, probably due to lack of adequate resolution. The observation of three well resolved components for the $J=1$ state is consistent with a site symmetry of $C_{2 y}$ or lower.

The site models illustrated in Fig. $4 b-d$ in which each $\mathrm{RE}^{3+}$ cation is adjacent to either a vacancy or another $\mathrm{RE}^{3+}$ cation exhibit $\mathrm{C}_{2}$ site symmetry. The local coordination geometry for the defect configurations illustrated are therefore consistent with both the observed crystal field splitting and the requirements for charge neutrality.

The dipole selection rules for RE cations with half-integral $J$-states are less restrictive than those for integral $J$ states. As a result, the spectra of the $\mathrm{Nd}^{3+}$ and $\mathrm{Sm}^{3+}$ intercalates are less diagnostic than those of the $\mathrm{Eu}^{3+}$ compound. Nevertheless, the PL spectra of the dehydrated phases of $\mathrm{CdPS}_{3}: \mathrm{Nd}^{3+}$ and $\mathrm{CdPS}_{3}: \mathrm{Sm}^{3+}$ (Fig 2a-c) are consistent with and support the analysis of the $\mathrm{Eu}^{3+}$ intercalate spectrum. For example, the $\mathrm{CdPS}_{3}: \mathrm{Nd}^{3+}{ }^{4} F_{3 / 2} \rightarrow{ }^{4} I_{9 / 2}$ transition is fully resolved into its five crystal field components as predicted for trigonal or lower site symmetry. The spectra of $\mathrm{Sm}^{3+}$ reveals the $J$-multiplet splitting of the ${ }^{6} \mathrm{H}_{5 / 2},{ }^{6} \mathrm{H}_{7 / 2}$ and ${ }^{6} \mathrm{H}_{9 / 2}$ manifolds (Fig. 3). In each case, the number of lines observed is equal to the number expected for trigonal or lower symmetry: three for the ${ }^{6} \mathrm{H}_{5 / 2}$ manifold, four for the ${ }^{6} \mathrm{H}_{7 / 2}$ manifold and five for the ${ }^{6} H_{9 / 2}$ manifold.

The hydrated phases either do not luminesce (Eu) or luminesce only weakly (Nd, $\mathrm{Sm}$ ). This behaviour is typical of RE cations when they are coordinated by water [19]. In addition, the spectra obtained from the hydrated CdPS $_{3}:$ RE $^{3+}$ phases exhibit only broad, poorly resolved structure. For example, the crystal field splitting of the $\mathrm{Sm}^{3+6} \mathrm{H}_{7 / 2}$ manifold observed in the hydrated phase is compared with that obtained from the dehydrated phase in Fig. 5. As expected, the changed coordination environment only slightly shifts the position of the multiplet. However, the luminescence intensity is dramatically decreased, as evidenced by the poorer signal-to-noise ratio, and the linewidth is approximately doubled. The line broadening is likely the result of the inhomogeneity of the vWG. Additionally, the manifold-wide splitting is decreased by about $25 \%$ reflecting the reduced strength of the crystal field experienced by solvated cations within the vWG. The number of crystal field components, however, is unchanged, suggesting that

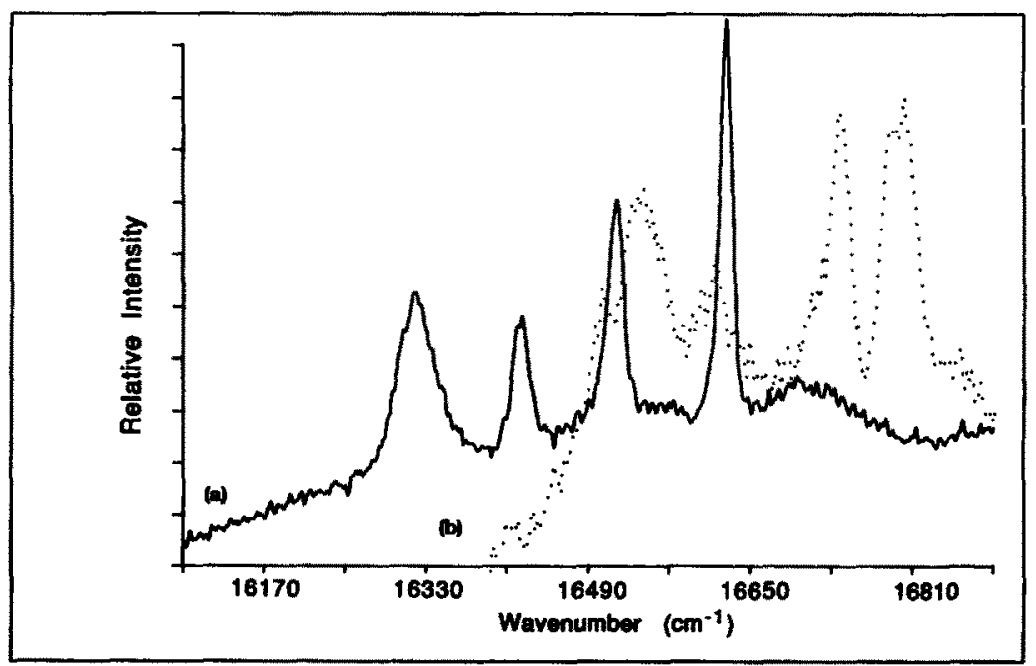

Fig. 5. Comparison of the $\mathrm{Sm}^{3+4} G_{5 / 2} \rightarrow{ }^{6} H_{7 / 2}$ multiplet in the (a) hydrated and (b) dehydrated phase. 
the coordination geometry in the vWG is trigonal or lower. This is the result anticipated for the solvated $\mathrm{RE}^{3+}$ cation in the field of a lattice cation vacancy.

\section{Other lattice effects}

The increased linewidth of the $J$-multiplet levels with decreasing transition energy (Fig. 3) is a characteristic feature of RE spectra. The line coming from the lowest crystal field level of the term is always the sharpest and the linewidths of the transitions to the higher crystal field levels are progressively greater. The behaviour is due to the rapid relaxation of the higher crystal field levels to the lower ones via spontaneous phonon emission [20].

The line width and spectral splittings of the $4 f$ electrons should increase in order $\mathrm{Eu}^{3+} \rightarrow \mathrm{Sm}^{3+} \rightarrow$ $\mathrm{Nd}^{3+}$, while fluorescence intensity should decrease in the same order [21]. Such a trend is observed when comparing the observed photoluminescence spectra of $\mathrm{Eu}^{3+}, \mathrm{Sm}^{3+}$ and $\mathrm{Nd}^{3+}$ in $\mathrm{CdPS}_{3}$.

\section{CONCLUSIONS}

Analysis of the luminescence spectra of three $\mathrm{RE}^{3+}$ cations inserted into the lamellar host $\mathrm{CdPS}_{3}$ via cation exchange in aqueous media suggests that a single emissive site is present in each case. Based on the number of $J$-multiplet components observed in the emission spectrum of each ion and charge compensation requirements, the site must have $C_{2}$ or lower symmetry. The symmetry reduction probably results from the pairing of RE cation sites with other RE cations, with vacancies or with monovalent cations in order to preserve local charge neutrality.

Acknowledgements - The authors would like to acknowledge support from the National Science Foundation (DMR 8818371). International collaboration was greatly assisted by funds from the NATO research program.

\section{REFERENCES}

1. Hahn H., Klingen W. and Eulenberger G., Naturwissenschaften 55, 229 (1968); Klingen W., Eulenberger G. and Hahn H., Z. Anorg. Allg. Chem. 401, 97 (1973); Klingen W., Ott R. and Hahn H., Z. Anorg. Allg. Chem. 396, 271 (1973).

2. Brec R., Schleich D., Ouvard G., Louisy A. and Rouxel J., Inorg. Chem. 18, 1814 (1979).

3. Johnson J., Intercalation Chemistry (Edited by M. S. Whittingham and A. J. Jacobson), p. 267. Academic Press, New York (1982).

4. Clement R., J. C. S. Chem. Comm. 647 (1980).

5. Cleary D. A., Groh J., Lifshitz E. and Francis A. H., J. Phys. Chem. 92, 551 (1988).

6. Wright J. C. and Cirillo-Penn K. M., Phys. Rev. B. Cond. Mat. 41, 10799 (1990).

7. Taylor B. E., Steger J. and Wold A., J. Solid St. Chem. 7, 461 (1973).

8. Harmer A. L., Linz A. and Gabbe D. R., J. Phys. Chem. Solids 30, 1483 (1969).

9. Dieke G. H., Spectra and Energy Levels of the Rare Earth Ions in Crystals (Edited by H. M. Crosswhite and H. Crosswhite), p. 233. Interscience Publishers, New York (1968).

10. Cunha M. C. F., Brito H. F., Zinner L. B., Vicentini G. and Nascimento A. B., Coord. Chem. Rev. 119, 1 (1992).

11. Lerf A. and Schöllhorn R., Inorg. Chem. 16, 2950 (1977).

12. Watts R., J. Mat. Sci. 8, 1201 (1973); Watts R. K., Point Defects in Crystals, p. 116. John Wiley, New York (1977).

13. McPherson G. L. and Meyerson S. L., Chem. Phys. Lett. 476, 471 (1990); McPherson G. L. and Francis A. H., Phys. Rev. Lett. 41, 1681 (1978); Barthem R. B., Buisson R. and Cone R. L., J. Chem. Phys. 91, 627 (1989).

14. Cleary D. A., Lifshitz E. and Francis A. H., J. Lum. 35, 163 (1986).

15. Lacroix P., Audiere J. P. and Clement R., J. C. S. Chem. Commun. 536 (1989).

16. Adapted from Sinha S. P. and Butter E., Molecular Phys. 16, 285 (1969).

17. Ofelt G. S., J. Chem. Phys. 37, 511 (1962).

18. Cunha M. C. F., loc. cit.

19. Hüfner S., Optical Spectra of Transparent Rare Earth Compounds, p. 128. Academic Press, New York (1978).

20. Hüfner S., loc. cit.

21. Yashevich E., Spectra of the Rare Earths 508 (1969). 